\title{
Whole-body vibration can attenuate the deterioration of bone mass and trabecular bone microstructure in rats with spinal cord injury
}

\author{
A Minematsu ${ }^{1}$, Y Nishii ${ }^{1}, \mathrm{H}$ Imagita $^{1}$, D Takeshita ${ }^{2}$ and S Sakata ${ }^{3}$
}

Study design: Experimental, controlled study.

Objective: To examine the effects of whole-body vibration (WBV) on bone mass and trabecular bone microstructure (TBMS) during the early stage in juvenile rats with spinal cord injury $(\mathrm{SCl})$.

Setting: Studied at the Kio University in Japan.

Methods: Thirty-four 8-week-old male Wistar rats were divided into 3 groups: the SCI group, the sham-operation group (SHAM) and the SCl+WBV group. WBV started on the 8th day after SCl. After 1 or 2 weeks of WBV treatment, measurements of tissue mineral density, trabecular bone mineral content (BMC) and parameters of TBMS were obtained by scanning the proximal tibias with $x$-ray micro-computed tomography. Serum levels of osteocalcin (OC) and of tartrate-resistant acid phosphatase 5b (TRACP 5b) were measured with ELISA.

Results: BMC, volume bone mineral density, bone volume (BV), BV fraction (BV/tissue volume) and connectivity density (Conn.D) of TBMS parameters were significantly higher in SCl+WBV rats than in SCl rats after 2-week WBV. The BMC and BV/TV of bone mass index correlated well with Conn.D, suggesting the preservation of Conn.D. induced by WBV. SCl+WBV rats showed a decrease in serum OC after 1-week WBV, but a quick recovery from that after 2-week WBV. There was no difference in serum TRACP $5 b$ among the 3 groups throughout the experimental period.

Conclusion: WBV treatment could attenuate the bone deterioration that occurs during the early stage in juvenile rats with $\mathrm{SCl}$. In a clinic, this early WBV intervention may be an effective rehabilitation modality for preventing bone fragility in SCl patients.

Spinal Cord (2016) 54, 597-603; doi:10.1038/sc.2015.220; published online 22 December 2015

\section{INTRODUCTION}

Osteoporosis is a well-known complication of spinal cord injury (SCI). The mechanism of bone loss by SCI is complex and is constituted by multiple factors involving mechanical, neurovascular, hormonal and metabolic factors. ${ }^{1,2}$ Bone loss following SCI begins within the first 6 months after the injury, and stabilizes between 12 and 16 months. ${ }^{3}$ Bone loss is especially prominent in sublesional bones such as the distal femur and proximal tibia. ${ }^{4-8}$ In fact, bone mass in the distal femur and proximal tibia decreased by $51 \%$ and $70 \%$, respectively, within 1 year after the injury in SCI patients. ${ }^{6}$ These two bones were so fragile that even a weak physical impact could cause a fracture in SCI patients. ${ }^{7,8}$ Therefore, the frequency of fracture in SCI patients was about twice as high as that of healthy people. ${ }^{9}$ Edwards et al. ${ }^{10}$ showed that in the femur and tibia of acute SCI patients trabecular bone mineral content (BMC) was reduced by $3.1-4.4 \%$ per month and volumetric bone mineral density (BMD) by $2.7-4.7 \%$ per month within 4 months after the injury. In addition, cortical BMC and volumetric BMD were noticeably reduced, and furthermore bone strength indices were decreased. ${ }^{10}$ The incidence of SCI is higher in young people aged 15-24 years. ${ }^{11}$ Children (aged 5-13 years) with
SCI, like adult patients with SCI, had lower BMD in hip and knee bones, resulting in a higher risk of fractures at the lower extremities. ${ }^{12}$ In view of these facts, bone fragility and fracture risk appear to arise during the early stage in younger patients with SCI. Moreover, as the duration of SCI is prolonged, the hip BMD value decreases, ${ }^{13}$ and consequently the fracture risk of hip bone may rise. Therefore, special intervention for preventing fracture should be performed as soon as possible after injury.

Prevention of bone loss has been attempted in SCI patients. The preventive effects of non-pharmacological interventions utilizing standing, walking, functional electrical stimulus or ultrasound on bone loss remain controversial. ${ }^{1,9,14-16}$ The effects of such interventions differed depending on research conditions, including subjects, methods, devices and interventions; for example, passive physical activity by standing was ineffective, whereas muscle contraction by functional electrical stimulus was effective. ${ }^{1,9,14-16}$ Whole-body vibration (WBV) has been reported as a potent intervention for maintenance and/or enhancement of bone tissue in people with low levels of BMD, in elderly post-menopausal women and in adolescents. ${ }^{17-19}$ In SCI patients, a combination of standing

\footnotetext{
${ }^{1}$ Department of Physical Therapy, Faculty of Health Science, Kio University, Nara, Japan; ${ }^{2}$ Department of Artificial Organs, National Cerebral and Cardiovascular Center, Osaka, Japan and ${ }^{3}$ Department of Physiology, Nara Medical University, Nara, Japan

Correspondence: Professor A Minematsu, Department of Physical Therapy, Faculty of Health Science, Kio University, 4-4-2 Umaminaka, Koryo-cho, Kitakatsuragi-gun, 635-0832 Nara, Japan.

E-mail: a.minematsu@kio.ac.jp

Received 17 June 2015; revised 8 November 2015; accepted 12 November 2015; published online 22 December 2015
} 
and WBV increased BMD in the trunk and spine, ${ }^{20}$ whereas low-magnitude WBV improved the deterioration of neither the $\mathrm{BMD}$ in the distal femur nor the microstructure in the proximal tibia. $^{21}$ On the other hand, WBV improved the biomechanical properties of bones, histomorphometric parameters of the trabecular area and $\mathrm{BMC}$ in ovariectomized rats ${ }^{22,23}$ and also microarchitecture and macro-biomechanical parameters in the rats suspended from their tails. ${ }^{24}$ In addition, WBV preserved both BMD in cancellous bone and trabecular structure. ${ }^{25}$ In an SCI animal model, however, the effects of WBV on bone remain unclear. Bramlett et al. ${ }^{26}$ reported that low-intensity vibration (LIV), which started on the 28th day after SCI and continued for 35 days, improved selected bone markers, but not BMD of the femur and tibia, in 3-month-old SCI rats. Bone damages were found within 3 weeks after SCI in 6-week-old rats ${ }^{27}$ and were more severe in SCI rats than in ovariectomized or sciatic neurectomy rats at the age of 6 weeks. $^{28,29}$ In addition, we have reported that deterioration of bone occurs as early as 1 week after injury in 8-week-old rats with SCI. ${ }^{30}$ Thus, juvenile rats with SCI showed the unexpectedly rapid deterioration of bone as compared with SCI patients. Therefore, this SCI animal model using juvenile rats appears appropriate for exploring the potent intervention to prevent bone deterioration. The aim of this study was to examine the effects of WBV on bone mass and trabecular bone microstructure (TBMS) during the early stage in juvenile rats with SCI.

\section{MATERIALS AND METHODS}

This study was approved by the Committee for Research Facilities of Laboratory Animal Sciences in the Kio University and was performed in accordance with the Guide for the Care and Use of Laboratory Animals published by the US National Institutes of Health (NIH Publication No.85-23, revised in 1996).

\section{Animals \\ Seven-week-old male Wistar rats (Japan SLC, Hamamatsu, Japan) were housed in cages in a facility with controlled temperature $\left(23 \pm 2{ }^{\circ} \mathrm{C}\right)$, humidity $(55 \pm 5 \%)$ and a light-dark cycle ( $12 \mathrm{~h}: 12 \mathrm{~h})$. Rats were fed a standard rodent chow (CE-2; Clea Japan, Tokyo, Japan) and water ad libitum throughout the experiment. After acclimatization for a week, 24 rats (8 weeks old) were assigned to the SCI and SCI+WBV groups and 10 rats to the SHAM group. Rats were anesthetized by intraperitoneal injection of pentobarbital sodium $\left(40 \mathrm{mg} \mathrm{kg}^{-1}\right)$ for subsequent surgical procedures. The SCI and SCI+WBV groups underwent laminectomy and the lower thoracic spinal cord was surgically transected using micro-scissors, and the SHAM group underwent a sham operation, in which the lower thoracic spinal cord was left intact. All operated rats were kept on a warming pad until they awoke from the anesthesia, and were treated with a subcutaneous injection of enrofloxacin $\left(10 \mathrm{mg} \mathrm{kg}^{-1}\right)$. Manual bladder expression was performed for the SCI rats until spontaneous micturition was recovered.}

\section{Experimental design}

WBV started on the 8th day after the surgery. Half of the SCI rats $(n=12)$ underwent WBV (frequency $=25 \mathrm{~Hz}$ of, amplitude $=0.6 \mathrm{~mm}$ of, $20 \mathrm{~min}$ per day, 5 days per week) with a commercially available vibration device (Denkosha, Nagano, Japan) for 1 or 2 weeks (SCI+WBV group). The vibration direction was vertical and the calculated acceleration was $0.7 \mathrm{~g}{ }^{18}$ The remaining SCI rats (SCI group: $n=12)$ and SHAM rats $(n=10)$ did not undergo WBV.

After 1 or 2 weeks of WBV treatment, rats were anesthetized with intraperioneal injection of pentobarbital sodium and killed by exsanguination from the carotid arteries. Serum samples were obtained for biochemical analyses. Bilateral soleus and extensor digitorum longus were collected and weighed, and bilateral tibias were removed and stored in $70 \%$ ethanol until analysis for bone mass and TBMS.

\section{Analysis of tissue mineral density, BMC and TBMS}

Using x-ray micro-computed tomography (Micro-CT; Hitachi Medical Corporation, Tokyo, Japan), the proximal tibias were scanned at $60 \mathrm{kV}$, $100 \mu \mathrm{A}$, with a voxel size of $26.4 \mu \mathrm{m}$ in the high-definition mode. A BMD phantom was simultaneously scanned under the same scanning condition to obtain tissue mineral density, BMC and volume BMD (BMC/tissue volume). Scanned data were transmitted to a personal computer and TBMS of the region of interest was analyzed using the bone analysis software, TRI BON 3D (Ratoc System Engineering, Tokyo, Japan). The ROI was a $2 \mathrm{~mm}$-length portion of the tibial metaphysis, and the first slice was scanned $1 \mathrm{~mm}$ distal from the physeal-metaphyseal demarcation. Tissue volume (TV), bone volume (BV), BV fraction (BV/TV), trabecular thickness (Tb.Th), trabecular width (Tb.W), trabecular number (Tb.N), trabecular separation (Tb.Sp), connectivity density (Conn.D) and trabecular bone pattern factor (TBPf) were assessed as parameters of TBMS in the proximal tibia.

\section{Dry bone and ash weight measurements}

After TBMS analysis, the tibias were dehydrated in $100 \%$ ethanol for $48 \mathrm{~h}$ and then dried at $100^{\circ} \mathrm{C}$ for $24 \mathrm{~h}$ with a drying machine (Advantec, Tokyo, Japan) to obtain the data of dry bone weight. Finally, the tibias were burned to ash at $600{ }^{\circ} \mathrm{C}$ for $24 \mathrm{~h}$ in an electric furnace (Nitto Kagaku, Nagoya, Japan), and the obtained ash was weighed.

\section{Serum biochemical analyses}

Serum samples were stored at $-80^{\circ} \mathrm{C}$ until analyzed. Serum concentrations of calcium, inorganic phosphate, total protein, creatinine and alkaline phosphatase were determined. Serum levels of bone formation marker osteocalcin (OC) and of bone resorption marker tartrate-resistant acid phosphatase 5b (TRACP 5b) were measured with an osteocalcin EIA kit (Biomedical Technologies, Stoughton, MA, USA) and TRACP 5b ELISA kit (Immunodiagnostic Systems, Boldon, UK), respectively.

\section{Statistical analysis}

All values were expressed as mean \pm s.d. Overall difference among the SHAM, SCI and SCI+WBV groups was determined by the Kruskal-Wallis test, and the differences between individual groups were examined when the overall difference was significant. Pearson's correlation coefficient was used to determine the relationship between bone mass and TBMS parameters. All statistical analyses were performed using Excel Statistics software (Excel 2012 version 1.08 for Windows; Social Survey Research Information, Tokyo, Japan). A $P$-value $<0.05$ was considered statistically significant.

\section{RESULTS}

Body weight, dry bone weight, ash weight and muscle weight Body weight, dry bone weight, ash weight and muscle (soleus and extensor digitorum longus) weight were significantly lighter in the SCI and SCI+WBV groups than in the SHAM group after WBV treatment for 1 or 2 weeks (Table 1). However, ash weight and muscle (soleus and extensor digitorum longus) weight were significantly heavier in the SCI+WBV group than in the SCI group after 1 week of WBV treatment (Table 1).

\section{Bone mass and TBMS parameters of the proximal tibia}

Tissue mineral density of the proximal tibia after 1 week of WBV treatment was significantly lower in the SCI group than in the SHAM and SCI+WBV groups, whereas the tissue mineral density after 2 weeks of WBV was significantly higher in the SCI+WBV group than in the SHAM group (Table 2). In contrast, BMC, volume BMD, $\mathrm{BV}$ and BV/TV were significantly lower in the SCI and SCI+WBV groups than in the SHAM group regardless of the WBV treatment period, but were significantly higher in the SCI+WBV group than in the SCI group (Table 2). These results were further confirmed by the proximal tibia tomograms of the 3 groups (Figure 1). 
Table 1 Final body weight, dry bone weight, ash weight and muscle weight

\begin{tabular}{|c|c|c|c|c|c|c|}
\hline & \multicolumn{3}{|c|}{1 week } & \multicolumn{3}{|c|}{2 weeks } \\
\hline & SHAM & $\mathrm{SCl}$ & $S C l+W B V$ & SHAM & $\mathrm{SCl}$ & $S C l+W B V$ \\
\hline Dry bone weight (mg) & $349.9 \pm 18.9$ & $296.3 \pm 28.5^{b}$ & $314.9 \pm 10.9^{b}$ & $381.0 \pm 16.8$ & $328.8 \pm 18.2^{\mathrm{b}}$ & $324.0 \pm 17.5^{b}$ \\
\hline Ash weight (mg) & $201.7 \pm 10.1$ & $168.9 \pm 15.5^{b}$ & $179.5 \pm 7.6^{\mathrm{b}, \mathrm{c}}$ & $222.6 \pm 9.0$ & $187.7 \pm 10.2^{b}$ & $184.3 \pm 10.3^{b}$ \\
\hline Soleus weight (mg) & $94.9 \pm 10.0$ & $41.8 \pm 6.4^{\mathrm{b}}$ & $65.5 \pm 22.5^{\mathrm{a}, \mathrm{d}}$ & $106.1 \pm 8.0$ & $56.2 \pm 22.7^{b}$ & $68.4 \pm 26.5^{b}$ \\
\hline
\end{tabular}

Abbreviations: EDL, extensor digitorum longus; SCI, spinal cord injury; WBV, whole-body vibration.

asignificantly different from the SHAM group $(P<0.05)$

bignificantly different from the SHAM group $(P<0.01)$

'Significantly different from the SCl group $(P<0.05)$. Means \pm s.d.

dSignificantly different from the SCl group $(P<0.01)$

Table 2 Bone mass and trabecular bone microstructure parameters in the proximal tibia

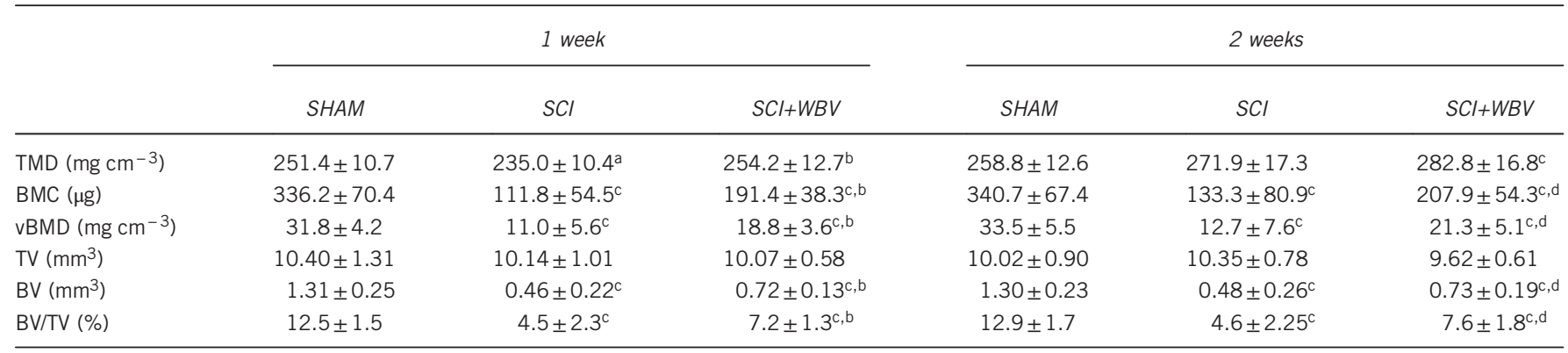

Abbreviations: BMC, bone mineral content; BV, bone volume; SCI, spinal cord injury; TMD, tissue mineral density; TV, tissue volume; vBMD, volume bone mineral density; WBV, whole-body vibration.

asignificantly different from the SHAM group $(P<0.05)$

Significantly different from the SHAM group $(P<0.05)$
bSignificantly different from the SCI group $(P<0.01)$.

bignificantly different from the SCl group $(P<0.01)$.
cSignificantly different from the SHAM group $(P<0.01)$.

dSignificantly different from the SCI group $(P<0.05)$. Means \pm s.d.
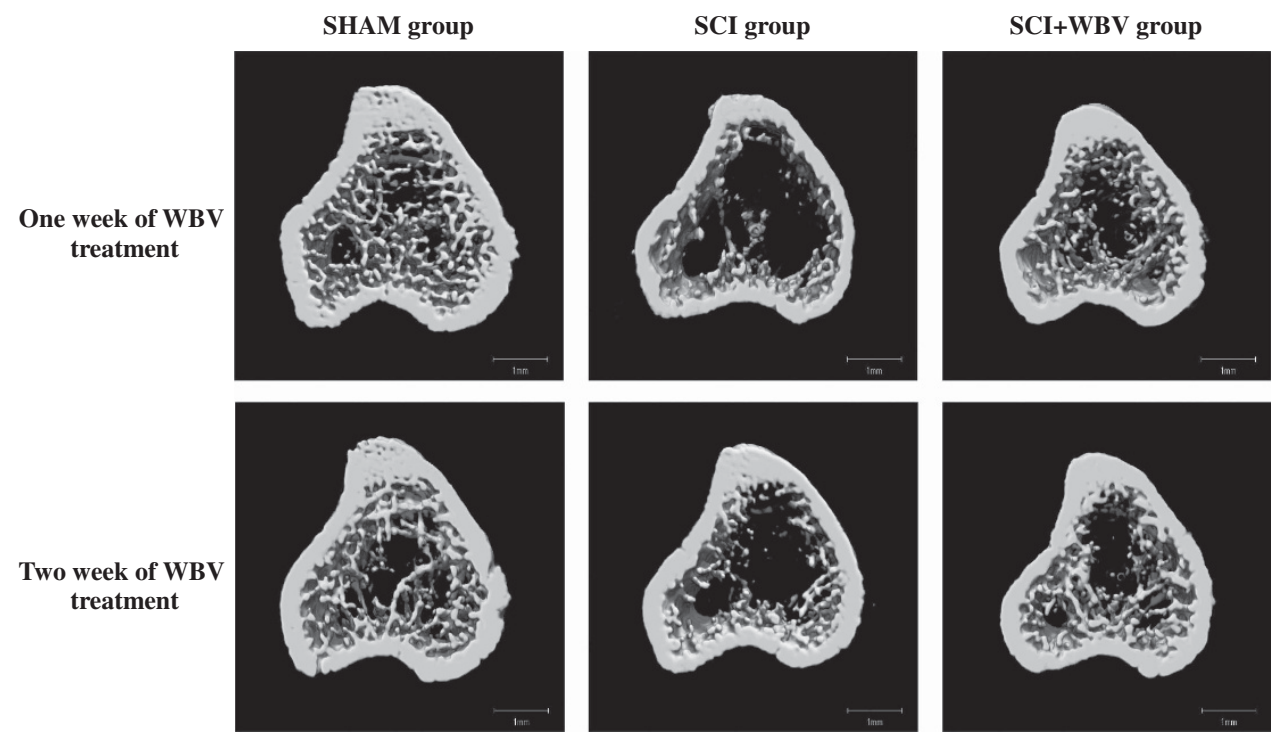

Figure 1 Cross sections of region of interest in the proximal tibia.

After 1 week of WBV treatment, Tb.Th, Tb.W, Tb.N and Conn.D were significantly lower in the SCI and SCI+WBV groups than in the SHAM group (Figure 2). However, Tb.N and Conn.D were significantly higher in the SCI+WBV group than in the SCI group. Tb. Sp was significantly higher in the SCI group than in the SHAM group, but Tb.Sp of the SCI+WBV group was similar to that of the SHAM group (Figure 2). TBPf was significantly higher in the SCI and SCI+WBV groups than in the SHAM group (Figure 2). After 2 weeks of WBV treatment, likewise, Tb.Th and Tb.W in the SCI group and Tb.N and Conn.D in the SCI and SCI+WBV groups were significantly 

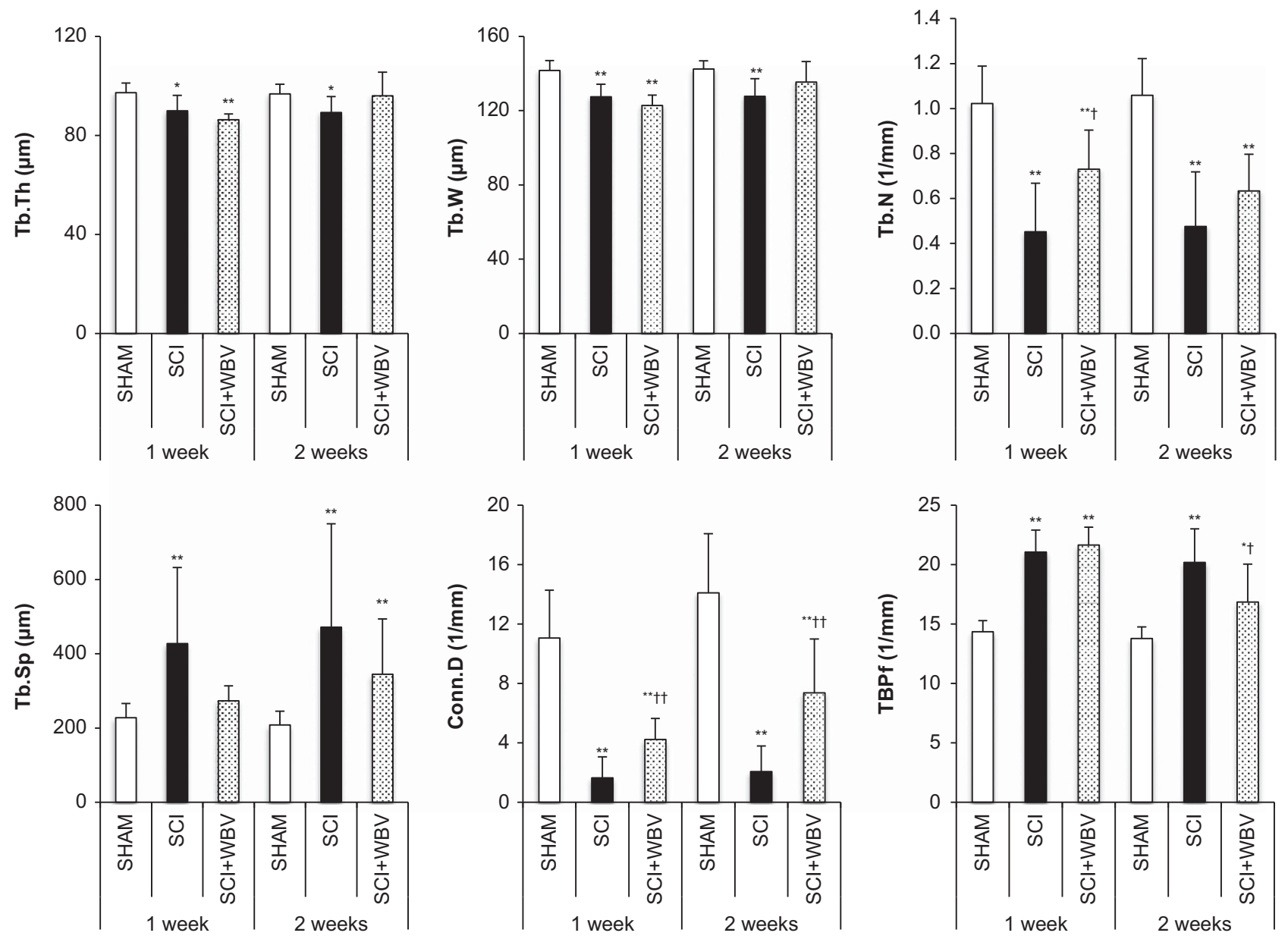

Figure 2 Parameters of trabecular bone microstructure in the proximal tibia. ${ }^{*}$ Significantly different from the SHAM group $(P<0.05)$. ${ }^{*}$ Significantly different from the SHAM group $(P<0.01)$. †Significantly different from the SCl group $(P<0.05)$. ††Significantly different from the $\mathrm{SCl}$ group $(P<0.01)$. Each vertical bar means s.d.

lower than those of the SHAM group (Figure 2). However, Conn.D of the SCI+WBV group was significantly higher than that of the SCI group (Figure 2). Tb.Sp was significantly higher in the SCI and SCI+WBV groups than in the SHAM group (Figure 2). TBPf was also significantly higher in the SCI and SCI+WBV groups than in the SHAM group, but significantly lower in the SCI+WBV group than in the SCI group (Figure 2).

\section{Serum biochemical analyses}

Serum creatinine concentrations were significantly lower in the SCI group than in the SHAM group after 1 week of WBV treatment (Table 3). After 2 weeks of WBV treatment, serum alkaline phosphatase concentrations were significantly higher in the SCI + WBV group than in the SHAM group (Table 3). Although serum OC levels of the SCI+WBV group, as well as of the SCI group, were significantly lower than those of the SHAM group after 1 week of WBV treatment, they reached the OC levels of the SHAM group after 2 weeks of WBV treatment (Figure 3). On the other hand, there was no significant difference in serum TRACP $5 \mathrm{~b}$ levels among the 3 groups throughout the experimental period (Figure 3).

\section{Correlation between bone mass and TBMS parameters}

Table 4 shows Pearson's correlation coefficients $(P<0.05)$ for bone mass and TBMS parameters obtained from the 3 groups. BV/TV correlated positively with Tb.Th, Tb.W, Tb.N and Conn.D, and negatively with Tb.Sp and TBPf. There were fairly good negative correlations between TBPf and Tb.Th, Tb.W or Conn.D.

\section{DISCUSSION}

In the present study, WBV during the early stage of SCI attenuated the deterioration of bone mass and TBMS that appears as early as 1 week after SCI. This finding suggests that WBV may be an effective therapeutic intervention for preventing bone loss in SCI patients.

In a transected SCI model rat aged 6 weeks, dry bone weight and ash weight in the femur and tibia decreased significantly within 3 weeks after SCI, and trabecular bone mass in the proximal tibia decreased to $60-80 \%$ compared with the SHAM controls. ${ }^{27}$ Moreover, we have reported that tibia bone mass and TBMS parameters deteriorate as early as 1 week after SCI in 8 -week-old rats. ${ }^{30}$ In a contusion SCI model, likewise, there was a $48 \%$ decrease in trabecular bone mass and a $35 \%$ decrease in cortical bone mass at the distal femoral metaphysis on the 10th day after SCI in 7-week-old rats. ${ }^{31}$ Thus, regardless of SCI model type, SCI caused bone loss and TBMS deterioration at the early stage of SCI in juvenile rats. Thus, the bones of growing rats were rapidly damaged by SCI. In the present study as well as in previous studies, ${ }^{27,30,31}$ bone mass, TBMS and muscle weight deteriorated remarkably during the early stage of SCI. In addition, such deterioration was found to be attenuated by WBV. However, this 
Table 3 Results of serum biochemical analyses

\begin{tabular}{|c|c|c|c|c|c|c|}
\hline & \multicolumn{3}{|c|}{1 week } & \multicolumn{3}{|c|}{2 weeks } \\
\hline & SHAM & $\mathrm{SCl}$ & $S C l+W B V$ & SHAM & $\mathrm{SCl}$ & $S C l+W B V$ \\
\hline Inorganic phosphate $\left(\mathrm{mg} \mathrm{dl}^{-1}\right)$ & $9.7 \pm 1.1$ & $8.9 \pm 0.5$ & $9.3 \pm 0.7$ & $8.5 \pm 0.4$ & $8.6 \pm 0.5$ & $8.5 \pm 0.4$ \\
\hline Total protein $\left(\mathrm{g} \mathrm{dl}^{-1}\right)$ & $5.7 \pm 0.2$ & $5.8 \pm 0.3$ & $5.8 \pm 0.3$ & $6.0 \pm 0.1$ & $5.9 \pm 0.4$ & $5.8 \pm 0.3$ \\
\hline Creatinine $\left(\mathrm{mg} \mathrm{dl}^{-1}\right)$ & $0.26 \pm 0.03$ & $0.20 \pm 0.02^{\mathrm{a}}$ & $0.24 \pm 0.04$ & $0.24 \pm 0.01$ & $0.23 \pm 0.02$ & $0.23 \pm 0.02$ \\
\hline
\end{tabular}

Abbreviations: $\mathrm{SCI}$, spinal cord injury; WBV, whole-body vibration.

asignificantly different from the SHAM group $(P<0.05)$. Mean \pm s.d.
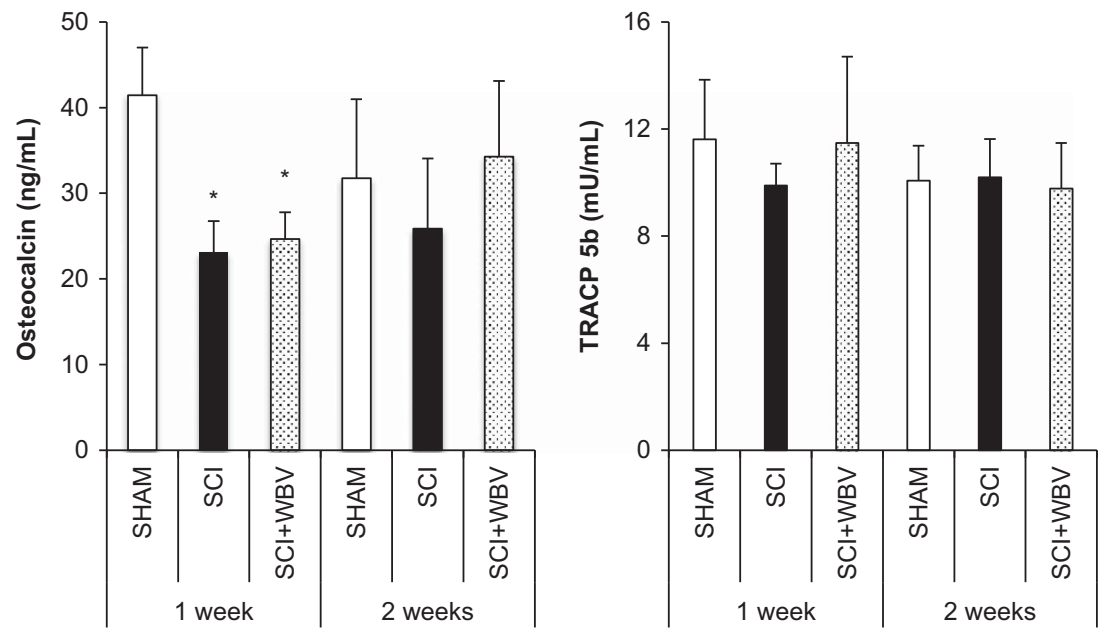

Figure 3 Serum osteocalcin and TRACP 5b levels. *Significantly different from the SHAM group $(P<0.05)$. Each vertical bar means s.d.

Table 4 Correlation between bone mass and trabecular bone microstructure parameters

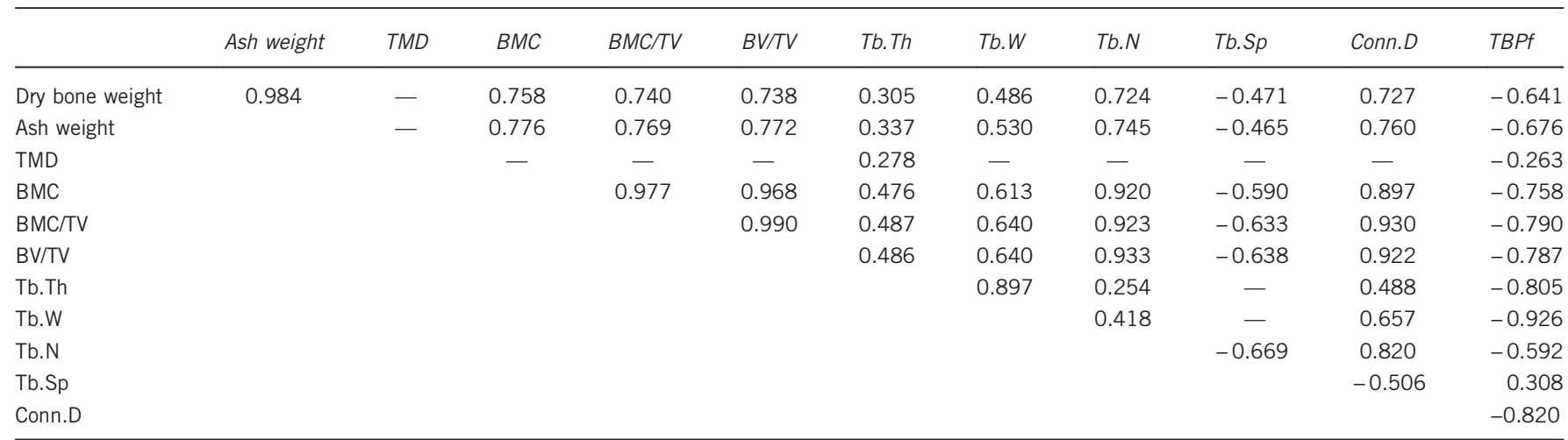

Abbreviations: BMC, bone mineral content; BMC/TV, BMC/tissue volume; BV/TV, bone volume/tissue volume; Conn.D, connectivity density; Tb.N, trabecular number; TBPf, trabecular bone pattern factor; Tb.Sp, trabecular separation; Tb.Th, trabecular thickness; Tb.W, trabecular width; TMD, tissue mineral density.

Data shows Pearson's correlation coefficients. - indicates no significance $(P>0.05)$.

WBV effect may be affected by the timing of initiating WBV treatment. Bramlett et al. ${ }^{26}$ showed that LIV for 35 days does not alter BMD and trabecular architecture in the contusion SCI model rats aged 3 months. In chronic SCI patients, either, LIV was not effective in improving BMD and TBMS. ${ }^{21}$ Moreover, in chronic SCI patients, daily standing $(>1 \mathrm{~h})$ showed no significant change in mean t-score of the proximal femur region. ${ }^{32}$ In acute SCI patients (8-12 weeks after SCI), however, passive standing ( $>1 \mathrm{~h}$ ) was capable of preserving bone mass and improving $\mathrm{BMD}$ as compared with those of non-standing patients ( $>2$ years after SCI). ${ }^{33}$ Thus, the mechanical loading interventions, such as vibration or weight-bearing, during the early stage of SCI appear potent for preventing bone loss and maintaining bone mass. In line with this idea, the present study shows that WBV, initiated on the 8th day after SCI, is effective in attenuating the deterioration in bone mass, TBMS and muscle weight. Bramlett et al. ${ }^{26}$, who started LIV on the 28th day after SCI, suggested 
that LIV, initiated earlier after SCI and/or continued for a longer period, may improve bone mass.

In the present study as well as in previous studies, ${ }^{28-30,34}$ dry bone weight, ash weight and BV/TV decreased, and TBMS parameters changed during the early stage of SCI in 6-8-week-old rats. However, the decrease in BMC, BV and BV/TV was significantly attenuated after 1 or 2 weeks of WBV treatment. The SCI rats revealed a $61-67 \%$ reduction in BMC, BV and BV/TV compared with the SHAM rats, whereas the SCI+WBV rats had a $39-45 \%$ reduction. The reduction in Conn.D after SCI was also attenuated by WBV treatment for 1 or 2 weeks. WBV treatment could also attenuate the deterioration of BV/TV, which strongly correlated with Conn.D. The inhibition of bone resorption and/or the acceleration of bone formation is necessary for preventing TBMS deterioration. The bone resorption markers increased in the acute stage and remained elevated in the chronic stage, whereas the circulating levels of OC increased or remained unchanged in the acute and chronic stages after SCI. ${ }^{35}$ Thus, rapid bone loss after SCI appears to be caused by the consistent increase in bone resorption. Bramlett et al. ${ }^{26}$ reported that serum levels of C-terminal telopeptide of type I collagen degradation products (CTX) increased significantly in the SCI and SCI+LIV groups compared with the SHAM group. On the other hand, serum OC levels were significantly higher in the SCI+LIV and SHAM groups than in the SCI group, ${ }^{26}$ suggesting that LIV can promote bone formation even after SCI. In the present study, although serum OC levels in the SCI and SCI+WBV groups decreased after 1 week of WBV treatment compared with the SHAM group, the OC levels of the SCI+WBV group reached the OC levels of the SHAM group after 2 weeks of WBV treatment. In addition, serum alkaline phosphatase levels were significantly higher in the SCI+WBV group than in the SHAM group after 2 weeks of WBV treatment. On the other hand, there was no difference in serum TRACP 5 b levels among the 3 groups throughout the experiment. Thus, the WBV-induced TBMS improvement would have resulted from the resumed bone formation and/or an excess of bone formation over bone resorption.

In our study, WVB could attenuate bone loss and deterioration during the short-term ( 1 or 2 weeks) treatment. Moreover, BMC and $\mathrm{BV} / \mathrm{TV}$ of bone mass index were found to correlate fairly well with $\mathrm{Tb}$. $\mathrm{N}$ and Conn.D of TBMS parameters. In addition, Conn.D correlated with Tb.N, Tb.Th and Tb.W. These results suggest that bone mass is closely linked to trabecular bone connectivity. WBV treatment is thought to attenuate the bone loss by preserving Conn.D, because it seems difficult to preserve the TBMS parameters other than Conn.D once trabecular bone is diminished. Therefore, it seems likely that $\mathrm{WBV}$ attenuates the SCI-induced $\mathrm{BV} / \mathrm{TV}$ reduction via an improvement in Conn.D.

The density of osteoclasts in cultured bone cells was much smaller in the SCI+LIV rats than in the SCI rats, and consequently serum CTX levels were significantly lower in the SCI+LIV rats than in the SCI rats. ${ }^{26}$ On the other hand, although serum OC levels were significantly higher in the SCI+LIV rats than in the SCI rats, there was no difference in the density of colony forming unit-fibroblast between the SCI and SCI+LIV rats. In addition to the measurement of bone metabolic markers, such a cell culture study is required to evaluate the effects of WBV on the bone formation and resorption and to clarify the physiological differences between the contusion and the transection models of SCI. Moreover, we will need to examine the effects of a longer period of WBV and/or a longer time of daily treatment on bone properties for exploring the most effective condition of WBV treatment.

\section{CONCLUSION}

On the basis of the above-mentioned results, we conclude that WBV treatment can attenuate the bone deterioration that occurs during the early stage in juvenile rats with SCI. In a clinic, this early WBV intervention may be an effective rehabilitation modality for preventing bone fragility in SCI patients. Further studies are required for examining the effects of WBV, which starts at various stages after SCI and/or continues for the longer period, on bone mass, TBMS and mechanical strength.

\section{DATA ARCHIVING}

There were no data to deposit.

\section{CONFLICT OF INTEREST}

The authors declare no conflict of interest.

1 Maimoun L, Fattal1 C, Micallef JP, Peruchon E, Rabischong P. Bone loss in spinal cord-injured patients: from physiopathology to therapy. Spinal Cord 2006; 44: 203-210.

2 Jiang SD, Jiang LS, Dai LY. Mechanisms of osteoporosis in spinal cord injury. Clin Endocrinol 2006; 65: 555-565.

3 Uebelhartl D, Demiaux-Domenech B, Rothl M, Chantrainel A. Bone metabolism in spinal cord injured individuals and in others who have prolonged immobilisation. A review. Paraplegia 1995; 33: 669-673.

4 Eser P, Frotzler A, Zehnder Y, Wick L, Knecht H, Denoth J et al. Relationship between the duration of paralysis and bone structure: a pQCT study of spinal cord injured individuals. Bone 2004; 34: 869-880.

5 Zehnder Y, Luthi M, Michel D, Knecht H, Perrelet R, Neto I et al. Long-term changes in bone metabolism, bone mineral density, quantitative ultrasound parameters, and fracture incidence after spinal cord injury: a cross-sectional observational study in 100 paraplegic men. Osteoporosis Int 2004; 15: 180-189.

6 Dauty M, Perrouin Verbe B, Maugars Y, Dubois C, Mathe JF. Supralesional and sublesional bone mineral density in spinal cord-injured patients. Bone 2000; 27: 305-309.

7 Gifre L, Vidal J, Carrasco J, Portell E, Puig J, Monegal A et al. Incidence of skeletal fractures after traumatic spinal cord injury: a 10-year follow-up study. Clin Rehabil 2014; 28: 361-369.

8 Vestergaard P, Krogh K, Rejnmark L, Mosekilde L. Fracture rates and risk factors for fractures in patients with spinal cord injury. Spinal Cord 1998; 36: 790-796.

9 Giangregorio L, McCartney N. Bone loss and muscle atrophy in spinal cord injury: epidemiology, fracture prediction, and rehabilitation strategies. J Spinal Cord Med 2006; 29: 489-500.

10 Edwards WB, Schnitzer TJ, Troy KL. Bone mineral and stiffness loss at the distal femur and proximal tibia in acute spinal cord injury. Osteoporosis Int 2014; 25: 1005-1015.

11 Acton PA, Farley T, Freni LW, Ilegbodu VA, Sniezek JE, Wohlleb JC. Traumatic spinal cord injury in Arkansa, 1980 to 1989. Arch Phys Med Rehabil 1993; 74: 1035-1040.

12 Lauer R, Johnston TE, Smith BT, Mulcahey MJ, Betz RR, Maurer AH. Bone mineral density of the hip and knee in children with spinal cord injury. J Spinal Cord Med 2007; 30: S10-S14.

13 Kaya K, Aybay C, Ozel S, Kutay N, Gokkaya O. Evaluation of bone mineral density in patients with spinal cord injury. J Spinal Cord Med 2006; 29: 396-401.

14 Biering-Sorensen F, Hansen B, Lee BSB. Non-pharmacological treatment and prevention of bone loss after spinal cord injury: a systematic review. Spinal Cord 2009; 47: 508-518.

15 Dolbow DR, Gorgey AS, Daniels JA, Adler RA, Moore JR, Gater DR Jr. The effects of spinal cord injury and exercise on bone mass: a literature review. Neuro Rehabil 2011; 29: 261-269.

16 Battaglino R, Lazzari A, Garshick E, Morse L. Spinal cord injury-induced osteoporosis: pathogenesis and emerging therapies. Curr Osteoporosis Rep 2012; 10: 278-285.

17 Prisby RD, Lafage-Proust MH, Malaval L, Belli A, Vico L. Effects of whole body vibration on the skeleton and other organ systems in man and animal models: what we know and what we need to know. Aging Res Rev 2008; 7: 319-329.

18 Totosy de Zepetnek JO, Giangregorio LM, Craven BC. Whole-body vibration as potential intervention for people with low bone mineral density and osteoporosis: a review. J Rehabil Res Dev 2009; 46: 529-542.

19 Slatkovska L, Alibhai SMH, Beyene J, Cheung AM. Effect of whole-body vibration on BMD: a systematic review and meta-analysis. Osteoporosis Int 2010; 21: 1969-1980.

20 Davis R, Sanborn C, Nichols D, Bazett-Jones DM, Dugan E. The effects of whole body vibration on bone mineral density for a person with a spinal cord injury: a case study. Adapt Phys Activ Q 2010; 27: 60-72.

21 Wuermser LA, Beck LA, Lamb JL, Atkinson EJ, Amin S. The effect of low-magnitude whole body vibration on bone density and microstructure in men and women with chronic motor complete paraplegia. J Spinal Cord Med 2015; 38: 178-186. 
22 Sehmisch S, Galal R, Kolios L, Tezval M, Dullin C, Zimmer S et al. Effects of low-magnitude, high-frequency mechanical stimulation in the rat osteopenia model. Osteoporos Int 2009; 20: 1999-2008.

23 Tezval M, Biblis M, Sehmisch S, Schmelz U, Kolios L, Rack T et al. Improvement of femoral bone quality after low-magnitude, high-frequency mechanical stimulation in the ovariectomized rat as an osteopenia model. Calcif Tissue Int 2011; 88: 33-40.

24 Zhang R, Gong H, Zhu D, Gao J, Fang J, Fan Y. Seven day insertion rest in whole body vibration improves multi-level bone quality in tail suspension rats. PLOS ONE 2014; 9: e92312.

$25 \mathrm{Li} \mathrm{Z,} \mathrm{Tan} \mathrm{C,} \mathrm{Wu} \mathrm{Y,} \mathrm{Ding} \mathrm{Y,} \mathrm{Wang} \mathrm{H,} \mathrm{Chen} \mathrm{W} \mathrm{et} \mathrm{al.} \mathrm{Whole-body} \mathrm{vibration} \mathrm{and} \mathrm{resistance}$ exercise prevent long-term hindlimb unloading-induced bone loss: independent and interactive effects. Eur J Appl Physiol 2012; 112: 3743-3753.

26 Bramlett HM, Dietrich WD, Marcillo A, Mawhinney LJ, Furones-Alonso O, Bregy A et al. Effects of low intensity vibration on bone and muscle in rats with spinal cord injury. Osteoporos Int 2014; 25: 2209-2219.

27 Jiang SD, Jiang LS, Dai LY. Changes in bone mass, bone structure, bone biomechanical properties, and bone metabolism after spinal cord injury: a 6-month longitudinal study in growing rats. Calcif Tissue Int 2007; 80: 167-175.

28 Jiang SD, Shen C, Jiang LS, Dai LY. Differences of bone mass and bone structure in osteopenic rat models caused by spinal cord injury and ovariectomy. Osteoporos Int 2007; 18: 743-750.
29 Jiang SD, Jiang LS, Dai LY. Spinal cord injury causes more damage to bone mass, bone structure, biomechanical properties and bone metabolism than sciatic neurectomy in young rats. Osteoporos Int 2006; 17: 1552-1561.

30 Minematsu A, Nishii Y, Imagita H, Sakata S. Time course changes in trabecular bone microstructure in rats with spinal cord injury. J Life Sci 2014; 8: $522-528$.

31 Morse L, Teng YD, Pham L, Newton K, Yu D, Liao WL et al. Spinal cord injury causes rapid osteoclastic resorption and growth plate abnormalities in growing rats (SCl-induced bone loss in growing rats). Osteoporos Int 2008; 19: 645-652.

32 Goktepe AS, Tugcu I, Yilmaz B, Alaca R, Gunduz S. Does standing protect bone density in patients with chronic spinal cord injury? J Spinal Cord Med 2008; 31: 197-201.

33 Alekna V, Tamulaitiene M, Sinevicius T, Juocevicius A. Effect of weight-bearing activities on bone mineral density in spinal cord injured patients during the period of the first two years. Spinal Cord 2008; 46: 727-732.

34 Liu D, Zhao CQ, Li H, Jiang SD, Jiang LS, Dai LY. Effects of spinal cord injury and hindlimb immobilization on sublesional and supralesional bones in young growing rats. Bone 2008; 43: 119-125.

35 Maïmoun L, Fattal C, Sultana C. Bone remodeling and calcium homeostasis in patients with spinal cord injury: a review. Metabolism 2011; 60 $1655-1663$. 